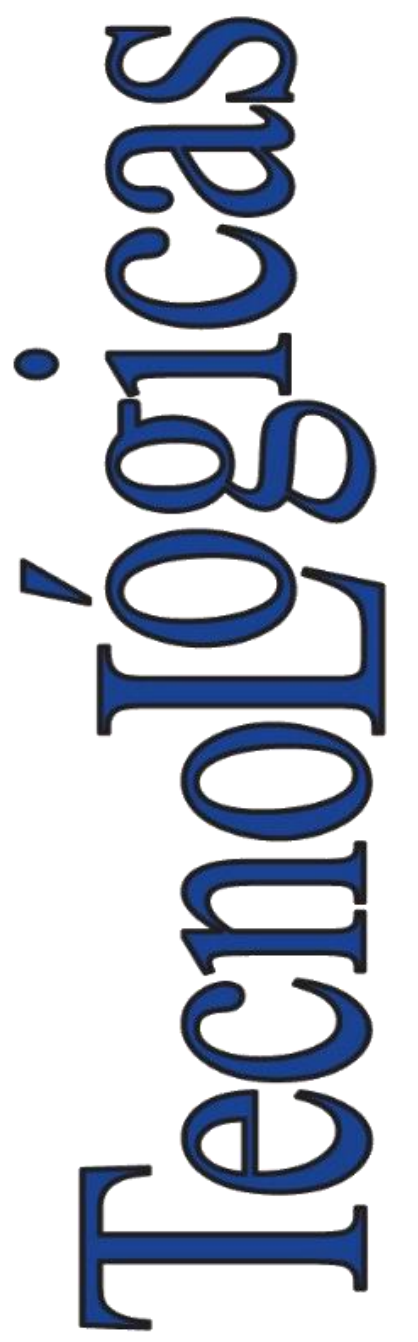

ISSN-p: 0123-7799 ISSN-e: $2256-5337$

Vol. 24, nro. 50, e1730

Recibido: 23 junio 2020 Aceptado: 11 noviembre 2020 Disponible: 18 diciembre 2020

CInstituto Tecnológico Metropolitano Este trabajo está licenciado bajo una Licencia Internacional Creative Commons Atribución (CC BY-NC-SA)

\section{Evaluación del efecto de la presencia de hongos patógenos y metabolitos secundarios sobre la germinación en tres hortalizas de hojas}

\section{Evaluation of the Effect of the Presence of Pathogenic Fungi and Secondary Metabolites on the Germination of Three Leaf Vegetables}

\author{
QSorianny Álvarez-Orozco ${ }^{1}$; \\ DDuilio Torres-Rodríguez ${ }^{2}$; \\ (DPastora Querales; \\ (DRosario Valera ${ }^{4}$; \\ DJosé Daniel Pacheco-Pacheco ${ }^{5}$; \\ DTatiana Gavilánez B. ${ }^{6}$
}

${ }^{1}$ Universidad Centroccidental Lisandro Alvarado, Cabudare-Venezuela, soriannyalvarezorozco@gmail.com

2 Universidad Centroccidental Lisandro Alvarado, Cabudare-Venezuela, duiliotr@yahoo.com

${ }^{3}$ Universidad Centroccidental Lisandro Alvarado, Cabudare-Venezuela, pastoraq@ucla.edu.ve

${ }^{4}$ Universidad Centroccidental Lisandro Alvarado, Cabudare-Venezuela, rosariovalera@ucla.edu.ve

${ }^{5}$ Universidad Centroccidental Lisandro Alvarado, Cabudare-Venezuela, jdpacheco2000@gmail.com

${ }^{6}$ Universidad Técnica de Cotopaxi. Ecuador, tatiana.gavilanez@utc.edu.ec

Cómo citar / How to cite

S. Álvarez-Orozco; D. Torres-Rodríguez; P. Querales; R. Valera; J. D. Pacheco-Pacheco; T. Gavilánez B., "Evaluación del efecto de la presencia de hongos patógenos y metabolitos secundarios sobre la germinación en tres hortalizas de hojas", TecnoLógicas, vol. 24, nro. 50, e1730, 2021. https://doi.org/10.22430/22565337.1730 


\title{
Resumen
}

Las semillas de hortalizas de hoja son más sensibles al ataque de patógenos, por lo que es necesario estudiar el potencial fisiológico de las mismas. Para ello, se evaluó la calidad fisiológica de semilla de acelga (Fordhook Giant), ajo porro (Large American Flag y American Flag) y espinaca (New Zealand y Viroflay). Para ello se determinó el porcentaje de germinación, porcentaje de emergencia, velocidad de emergencia, índice de velocidad de emergencia, microbiota y metabolitos secundarios por cromatografía de capa fina. Para las evaluaciones de germinación, emergencia e identificación de patógenos se realizaron 4 repeticiones usando 100 semillas por repetición. Los resultados muestran un bajo desempeño fisiológico en semillas de espinaca New Zealand y Ajo porro (Large American Flag y American Flag), lo que se vio reflejado en las variables debido a la presencia de hongos de los géneros Rhizopus, Fusarium, Penicillium, y las bacterias, que redujeron el vigor de las plántulas, así como un número importante de plántulas anormales, mientras que las semillas de espinaca Viroflay y Acelga Fordhook Giant presentaron una mejor germinación al primer conteo, sin embargo, los problemas relacionados con el vigor se reflejaron en las variables porcentaje de emergencia, velocidad de emergencia, índice de velocidad de emergencia, y con alto desarrollo de plántulas anormales, adicionalmente se detectó la presencia de los flavonoides el cual se asoció a una menor incidencia de Penicillium sp., en la semilla de espinaca viroflay aumentando la germinación en el primer conteo y la germinación total.

\section{Palabras clave}

Germinación, Metabolitos secundarios, plántulas, sanidad vegetal.

\begin{abstract}
Leaf vegetables are more sensitive to pathogen attack, so it is necessary to study the physiological potential of it. For this purpose, the physiological seed quality of chard (Fordhook Giant), garlic (Large American Flag and American Flag) and spinach (New Zealand and Viroflay) was evaluated. The germination rate, emergence rate, emergence velocity, emergence velocity index, microbiota and secondary metabolites were determined by thin layer chromatography. For the evaluations of germination, emergence and pathogen identification, 4 replicates were performed using 100 seeds per replicate. The results show a low physiological performance in seeds of New Zealand spinach and garlic pot (Large American Flag and American Flag), which was reflected in the variables due to the presence of fungi of the genera Rhizopus, Fusarium, Penicillium, and bacteria, which reduced the vigor of the seedlings, as well as a significant number of abnormal seedlings, while Viroflay and Fordhook Giant Chard spinach seeds presented a better germination at the first count, however, the problems related to vigor were reflected in the variables emergence rate, emergence velocity, emergence velocity index, and with high development of abnormal seedlings, additionally the presence of flavonoids was detected which was associated with a lower incidence of Penicillium sp., in Viroflay spinach seed increasing the germination at the first count and total germination.
\end{abstract}

\section{Keywords}

Germination, secondary metabolites, seedling, vegetal health. 


\section{INTRODUCCIÓN}

Las hortalizas de hojas como la acelga, la espinaca y el ajoporro tienen un alto valor nutricional debido a la alta concentración de vitaminas y minerales [1]. Por otro lado, al tener un alto contenido de antioxidantes [2], las hacen de una elevada demanda, ya que muchas de ellas, desde el punto de vista nutricional, se han asociadas a beneficios para la salud, al mejoramiento del sistema inmune [3], a la prevención de enfermedades cardiovasculares [4] y a la disminución del riesgo de cáncer [5].

La producción de estas hortalizas demanda elevados requerimientos de agua y nutrientes [6], así como condiciones edafoclimáticas particulares, como suelos con alta fertilidad y temperaturas que no superen los $20^{\circ} \mathrm{C}$ [7]. Debido a que su desarrollo se da en condiciones de bajas temperaturas y humedad relativamente alta, las mismas van estar sometidas a una serie de patógenos que pueden afectar su productividad, particularmente si atacan el área foliar [8].

La incidencia de patógenos no solo está asociada a las condiciones climáticas y al manejo fitosanitario preventivo que se pueda aplicar para reducir la infestación por debajo del umbral económico [9], sino a que también se deben reducir los posibles medios de trasmisión, como son los abonos orgánicos, el agua y las semillas [10].

En el caso de las semillas, los productores deben contar con materiales de alta calidad que garanticen una adecuada germinación y emergencia, y que los mismos estén libres de patógenos que puedan afectar el desarrollo de las plántulas y posteriormente el crecimiento vegetativo y reproductivo de las hortalizas, afectando, obviamente, su rendimiento y los beneficios económicos de los productores [10], [11].

Dentro de los costos de producción, las semillas representan uno de los conceptos más importantes, y en muchos países con bajo nivel tecnológico para la producción agrícola, este componente depende de la importación de material vegetal [12] [13]. Dada la inversión y los riesgos asociados en la adquisición de semillas, es necesario que esos insumos sean de alta calidad, de tal manera que se garantice que estén por encima de los estándares internacionales, requiriendo, en el caso de las hortalizas de hoja, que el porcentaje de germinación esté arriba del 80 \% [14], [15].

Para garantizar una adecuada germinación y emergencia de las semillas es indispensable que las condiciones de almacenamiento [16] garanticen adecuadas condiciones de temperatura y humedad [17] para reducir la aparición frecuente de patógenos. Además de las condiciones de almacenamiento, se ha reportado que las semillas poseen componentes como los metabolitos secundarios que le ofrecen resistencia a las semillas frente a la presencia de plagas y enfermedades, por lo que se deben seleccionar materiales con alto contenido de flavonoides y fenoles en las semillas o hacer pretratamientos con sustancias que las provean de estos componentes [18].

El objetivo de esa investigación fue evaluar el desempeño fisiológico de acelga (Fordhook Giant), ajoporros (Large American Flag y American Flag) y espinacas (New Zealand y Viroflay) mediante pruebas de germinación y el estado fitosanitario a través de la identificación de la microbiota con potencial patógeno. Adicionalmente, se determinaron los metabolitos secundarios, estableciendo su asociación con la sanidad de las semillas. 


\section{MATERIALES Y MÉTODOS}

\subsection{Ubicación geográfica}

Los diferentes ensayos se realizaron en diciembre del 2019 en los Laboratorios de Micología y Microtecnia, ubicados en las instalaciones de Posgrado de Agronomía de la Universidad Centroccidental Lisandro Alvarado (UCLA), Cabudare, Municipio Palavecino del estado Lara, Venezuela, a 1001'30”LN, 69¹6’30” LO y altitud de 495 msnm.

\subsection{Material experimental}

Se utilizaron híbridos de acelga (Fordhook Giant), ajoporros (Large American Flag y American Flag) y espinacas (New Zealand y Viroflay), los cuales fueron empacados en octubre de 2016, febrero de 2017, noviembre de 2016 y octubre de 2016, respectivamente.

\subsection{Prueba de germinación preliminar}

La selección del material experimental usado en el ensayo se realizó en el centro occidente venezolano después un estudio preliminar de 36 rubros de hortalizas de hojas utilizadas en semillas con tiempo de almacenamiento variable. El porcentaje de germinación en algunos casos fue alto y, en otros, bajo, lo que obligó a evaluaciones posteriores en aquellas semillas de bajo poder germinativo (Tabla 1).

Tabla 1. Germinación de rubros de hortalizas comercializados en la región Centroccidental de Venezuela. Fuente: elaboración propia.

\begin{tabular}{|c|c|}
\hline Grupo & Híbridos -\% de germinación \\
\hline $\begin{array}{l}1 \text { Porcentaje de germinación } \\
\qquad>90 \%\end{array}$ & $\begin{array}{l}\text { Brócoli (Cacique, Chief y Corato). } 100 \text { \%; Lechuga Tasela } 100 \% \text {; Lechuga } \\
\text { Romana Nader } 100 \% \text {; Cebolla - Yellow Granex Prr Hy } 100 \% \text {; Cebolla En } \\
\text { Rama (Frangant e Ikako) } 96 \% \text {; Lechuga - Black Seeded Simpson } 96 \% \text {; } \\
\text { Tomate - Aegean } 96 \%\end{array}$ \\
\hline $\begin{array}{r}2 \text { Porcentaje d } \\
\text { entre } 7\end{array}$ & $\begin{array}{l}\text { Colinabo - Oasis } 88 \% \text {; Pepino - Poinsett } 7688 \% \text {; Remolacha - Early } \\
\text { Wonder Tall Top } 88 \% \text {; Coliflor Synergy 84\%; Rabano Rubi } 84 \% \text {; Repollo } \\
\text { - Green Ball } 84 \% \text {; Lechuga - Michihili } 84 \% \text { : Cebolla - Yellow Granex Prr } \\
\text { Hy } 82 \% \text {; Zanahoria - Condor } 72 \%\end{array}$ \\
\hline $\begin{array}{c}3 \text { Porcentaje de germinación } \\
\text { entre } 50 \text { y } 70 \%\end{array}$ & $\begin{array}{l}\text { Lechuga - Great Lakes } 65968 \text { \%; Pepino - Marketmore } 64 \% \text {; Repollo - } \\
\text { Merlot 64\%; Tomate - Río Grande 64 \%; Cebolla - Texas } 438 \text { 60\%; Cebolla } \\
\text { - Texas Early Grano } 502 \text { Prr1 56\% }\end{array}$ \\
\hline $\begin{array}{l}4 \text { Porcentaje de germinación } \\
\qquad 50 \%\end{array}$ & $\begin{array}{l}\text { Repollo - Scarlet } 48 \text { \%; Acelga - Fordhook Giant } 24 \% \text {; Ajoporro - Large } \\
\text { American Flag } 20 \% \text {; Cebolla - Sangre De Toro } 16 \% \text {; Ajoporro - American } \\
\text { Flag } 12 \% \text {; Espinaca - Viroflay } 12 \% \text {; Pimentón - California } 4 \% \text {; } \\
\text { Pimentón - Yolo Wonder Mejorado } 4 \% \text {; Celery - Tall Utah 52-70 } 0 \text {; } \\
\text { Pimentón - Robur } 0 \% \text {; Espinaca - New Zealand 0 \%; Cilantro - Dark } \\
\text { Green Italian } 0 \%\end{array}$ \\
\hline
\end{tabular}

\subsection{Condiciones de almacenamiento}

La calidad de la semilla pudo ser afectada por las condiciones de almacenamiento, en particular por la fecha de empacado y de almacenamiento de los materiales utilizados en el ensayo, las cuales se pueden apreciar en la Tabla 2. 
Tabla 2. Fecha de empacado, tiempo de almacenamiento y condiciones de humedad semillas de acelga, ajo porro y espinaca comercializados en región Centroccidental de Venezuela. Fuente: elaboración propia.

\begin{tabular}{ccc}
\hline Híbrido & Fecha de empacado & Tiempo de almacenamiento \\
\hline Espinaca viroflay & Octubre 2016 & 37 meses \\
Espinaca New Zealand & Octubre-2016 & 37 meses \\
Ajoporro American Flag & Noviembre-2016 & 38 meses \\
& Febrero-2016 & 29 meses \\
Acelga Fordhook Giant & Octubre-2016 & 37 meses \\
\hline
\end{tabular}

\subsection{Prueba de germinación estándar}

La capacidad de germinación se evaluó mediante la prueba de (GE) que se realizó de acuerdo con los procedimientos establecidos por la International Seed Testing Association ISTA (2016). Para ello se dispuso de 400 semillas de cada lote en bandejas de plástico transparentes, y en su interior se puso papel absorbente previamente humedecido y esterilizado. En cada lote se distribuyeron cuatro bandejas dentro de una cámara de germinación a una temperatura aproximada de $26 \pm 2{ }^{\circ} \mathrm{C}$, con ciclos alternados de luz (12 h) y oscuridad (12 h), los conteos se realizaron a los 6 y 12 días, respectivamente, considerando como germinadas sólo las plántulas normales, o aquellas que no superaran el 50 \% de manchas necróticas. Las plántulas que no germinaron se consideraron como semillas sin germinar y las que superaban el 50 \% de manchas necróticas o no terminaban el proceso germinativo, se consideraban plántulas anormales.

\subsection{Ensayo topográfico al tetrazolio}

Para evaluar la viabilidad de las semillas, se realizó el ensayo topográfico al tetrazolio, el cual se llevó a cabo de acuerdo con lo establecido por el ISTA (2016), usando una solución acuosa de cloruro de 2,3,5-trifeniltetrazolio al 1,0\%; 100 por tratamiento se sumergieron en la solución. Las semillas que presentaron tinción menor a las 3/4 de las estructuras esenciales para el desarrollo germinativo, se consideraron semillas no viables, y aquellas que presentaron tinciones mayores, se consideraron semillas viables.

\subsection{Primer conteo de germinación}

En el primer conteo se procedió a evaluar las semillas que ya habían germinado. Para ello se registraron y se sacaron de la bandeja de germinación las semillas que ya habían cumplido su fase germinativa, mientras que el resto de las semillas se mantuvieron intactas.

\subsection{Porcentaje de emergencia (PE)}

Se realizó bajo condiciones de temperatura ambiente promedio de $28 \pm 2{ }^{\circ} \mathrm{C}$, utilizando arena de río como sustrato previamente esterilizada en autoclave. Se sembraron 400 semillas de cada lote, distribuyéndolas en 4 repeticiones de 100 semillas a una profundidad de $0,8 \mathrm{~cm}$, con una distancia entre semillas de $2,5 \mathrm{~cm}$, aproximadamente. Las semillas se colocaron en bandejas plásticas germinadoras de 200 alveolos (una semilla por alveolo), dispuestas de manera aleatoria. La emergencia de plantas normales fue evaluada diariamente considerando aquellas donde se observó la extensión de las primeras hojas verdaderas, considerando este día como el inicio de la emergencia. El conteo finaliza para cada repetición cuando durante tres días consecutivos la emergencia se hace constante. 


\subsection{Velocidad de emergencia (VE)}

Para el cálculo de VE se eligió (1) propuesta por [19]:

$$
\mathrm{VE}=\frac{(\mathrm{N} 1 \mathrm{E} 1)+(\mathrm{N} 2 \mathrm{E} 2)+\cdots+(\mathrm{Nn} \mathrm{En})}{\mathrm{E} 1+\mathrm{E} 2+\cdots+\mathrm{En}}
$$

Dónde: $\mathrm{VE}$ = velocidad de emergencia (días); $\mathrm{E}$ = número de plántulas emergidas en cada repetición; $\mathrm{N}$ = número de días para cada contaje.

Para el cálculo del Índice de velocidad de emergencia (IVE) se tomaron los resultados obtenidos en la evaluación diaria de porcentaje de emergencia, de acuerdo con (2) propuesta por [20]:

$$
\operatorname{IVE}=\frac{\mathrm{E} 1}{\mathrm{~N} 1}+\frac{\mathrm{E} 2}{\mathrm{~N} 2}+\cdots+\frac{\mathrm{En}}{\mathrm{Nn}}
$$

Dónde: IVE = Índice de velocidad de emergencia; $\mathrm{E}=$ número de plántulas emergidas en cada repetición; $\mathrm{N}$ = número de días de cada contaje.

\subsection{Contenido de humedad en semilla}

El método empleado fue el descrito en las Normas [14]. Para realizar este ensayo se tomaron $10 \mathrm{~g}$ de semillas de cada material y se tomó el peso inicial. Las semillas se secaron durante $17 \mathrm{~h}$ a $105^{\circ} \mathrm{C}$ y se enfriaron en un desecador antes de pesar. El contenido de humedad en porcentaje por peso se calcula con (3) [14]:

$$
M(\%)=\frac{\text { Pérdidad de peso }}{\text { Peso inicial }} \times 100=\frac{M 2-M 3}{M 2-M 1} \times 100
$$

Dónde: M1 =peso (g) del contenedor y tapa; M2 = peso (g) del contenedor, la tapa y el contenido antes del secado; M3 = peso (g) del contenedor, la tapa y el contenido después del secado

\subsection{Identificación del microbiota asociada a semillas y su relación con el potencial fisiológico}

El ensayo consistió en colocar cuatro repeticiones de 100 semillas de acelga, ajoporro y espinaca en bandejas plásticas rectangulares, dotadas interiormente de esponjas humedecidas con agua destilada esterilizada, sobre estas se colocaron gradillas rectangulares que soportaban tres hojas de papel absorbente - también humedecido - siguiendo las sugerencias de [21] y modificado en el laboratorio de micología. Todos los materiales utilizados en esta prueba fueron previamente esterilizados y las semillas previamente lavadas.

Las bandejas fueron incubadas a temperatura ambiente dentro de una cámara de crecimiento con fotoperiodo de 12 horas durante 9 días. Transcurrido el periodo de incubación, se procedió a la evaluación que consistió en observar, a través de lupa estereoscópica, el desarrollo de hongos y bacterias y, en microscopio óptico para determinar los géneros de hongos que se encontraban asociados a cada semilla. Para la determinación de géneros de hongos se contó con el apoyo bibliográfico y de los expertos. 


\subsection{Cuantificación de metabolitos secundarios en extractos de etanol}

Para la preparación de los extractos etanólicos (EE) se tomó 1 g de semillas de las variedades estudiadas, se colocaron en viales (recipientes) con alcohol etílico al $96 \%$ y se dejaron macerando por 15 días a temperatura ambiente. La determinación de alcaloides, flavonoides y fenoles en los EE se realizó mediante cromatografía de capa fina, utilizando cromatofolios de papel filtro cortados con dimensiones de $6,5 \mathrm{~cm}$ de largo por 2,5 cm de ancho.

Según [22], los solventes utilizados para cada grupo de metabolito secundario fueron, para alcaloides N-butanol: ácido acético y agua; para flavonoides benceno: ácido acético y agua; y para fenoles ácido acético: agua en proporciones 9:2:1, 12:7:2 y 1:9, respectivamente.

\subsection{Análisis estadístico}

Se empleó el programa estadístico InfoStat 2019 para realizar el análisis de varianza de los resultados de las variables estudiadas, y en aquellos casos que fue necesario, se hizo la prueba de medias de rangos múltiples de Tukey $(\mathrm{P}<0,05)$, y los resultados correspondientes a la determinación de la frecuencia de patógenos asociados a la semilla de cebolla fueron analizados mediante la prueba no paramétrica de Kruskal \& Wallis, por no cumplir con la homogeneidad de las varianzas. Del mismo modo, los resultados de frecuencia de patógenos asociados a la semilla de acelga, ajoporro y espinaca, fueron corregidos por Kruskal \& Wallis por falta de homogeneidad de las varianzas y exceso de variación para determinados patógenos. También se realizó un análisis multivariado para comparar la correlación entre todas las variables a través de los componentes principales, así como un análisis de correlación para estudiar la asociación entre el tiempo de empacado y el porcentaje de germinación.

\section{RESULTADOS Y DISCUSIÓN}

Los resultados de la evaluación de 36 híbridos de hortalizas comercializados en la región Centooccidental de Venezuela se muestran en la Figura 1. Allí se puede observar que el $57,14 \%$ presentaron una germinación optima (>90\%); 28,67 \%, germinación moderada (50$90 \%)$; y 14,29 \% fue deficiente (<50\%). Particular interés debe prestarse a los híbridos de espinacas Viroflay y New Zealand, ajoporros American Flag y Large American Flag y acelga Fordhook Giant, las cuales presentaron una germinación debido a la perdida de la viabilidad de las semillas por el tiempo de almacenamiento y proliferación de patógenos por posibles condiciones inadecuadas de almacenamiento [23].

Dado que el tiempo de empacado pudo influir en la calidad de las semillas y su desempeño fisiológico, lo cual se reflejó en una menor viabilidad y porcentaje de germinación, se realizó una análisis de regresión entre el tiempo de empacado y el porcentaje de germinación, encontrando una relación negativa entre el tiempo de almacenamiento y la germinación, lo que demuestra que a mayor tiempo de empacado las semillas pierden su viabilidad [24] debido al ataque de patógenos y a las condiciones inadecuadas de almacenamiento (Figura 2). 


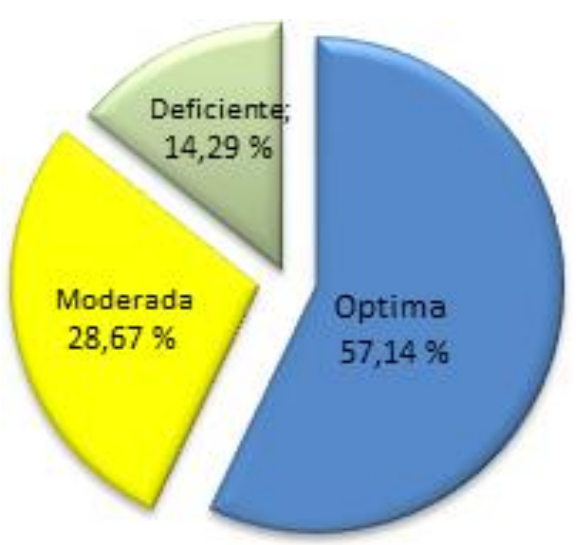

Figura 1. Porcentaje de germinación total de 36 híbridos de hortalizas comercializados en la región Centroccidental de Venezuela. Fuente: elaboración propia.

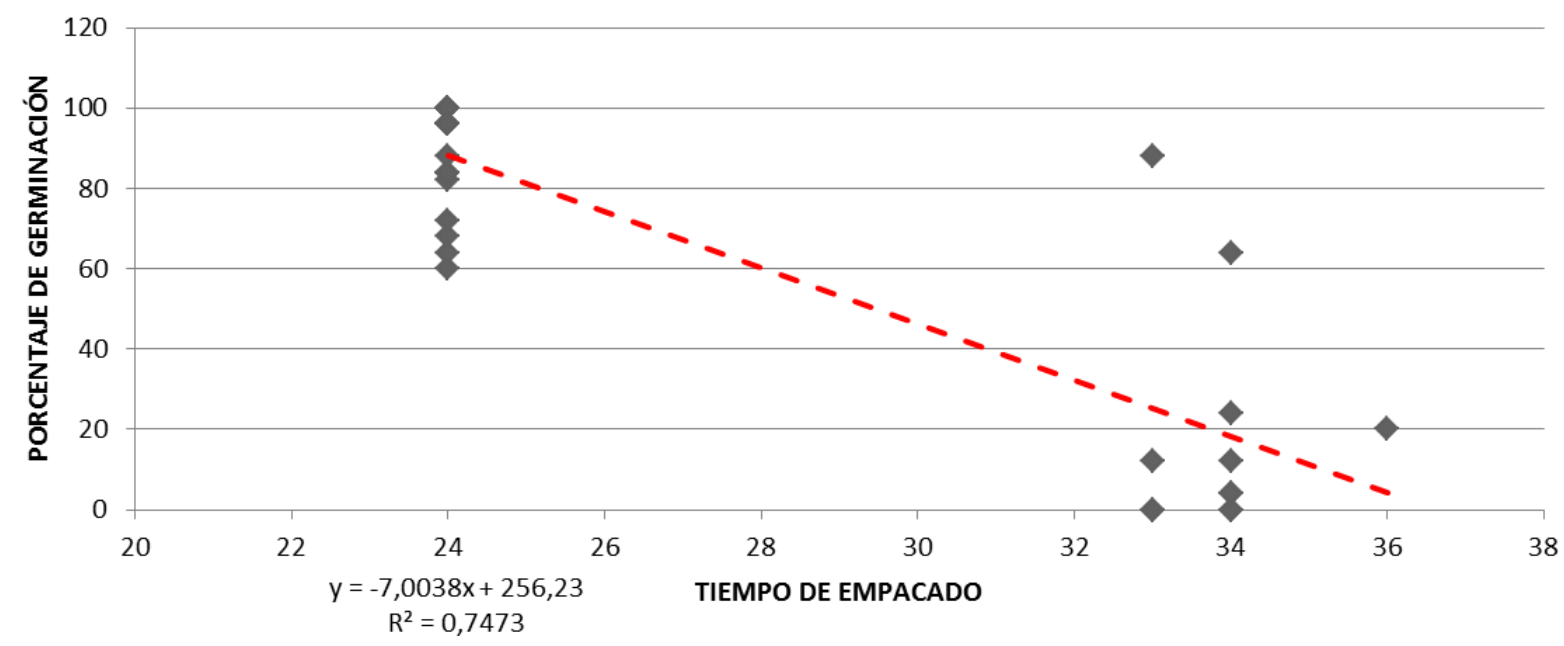

Figura 2. Relación tiempo de empacado de semillas de 36 rubros hortícolas comercializados en la región Centroccidental de Venezuela. Fuente: elaboración propia.

Se evaluó la germinación y el vigor de los cultivos de acelga, ajoporro y espinaca a través de las variables porcentaje de germinación (GE), germinación al primer contero (PC), porcentaje de emergencia (PE), velocidad de emergencia (VE) e índice de velocidad de emergencia (IVE), ver Tabla 3.

Tabla 3. Análisis de las variables de desempeño fisiológico de semillas de acelga, ajoporro y espinaca Fuente: elaboración propia.

\begin{tabular}{cccccc}
\hline Hortalizas & \multicolumn{4}{c}{ Vigor } \\
\cline { 2 - 6 } & GE (\%) & PC (\%) & PE & VE & IVE \\
\hline Espinaca viroflay & $66,00 \mathrm{c}$ & $29,50 \mathrm{c}$ & $19 \mathrm{~b}$ & $6,03 \mathrm{a}$ & $3,18 \mathrm{~b}$ \\
Espinaca New Zealand & $0,00 \mathrm{a}$ & $0,00 \mathrm{a}$ & $0 \mathrm{a}$ & $0,00 \mathrm{a}$ & $0,00 \mathrm{a}$ \\
Ajoporro American Flag & $9,00 \mathrm{a}$ & $0,00 \mathrm{a}$ & $2 \mathrm{a}$ & $5,25 \mathrm{a}$ & $0,16 \mathrm{a}$ \\
Ajoporro Large American Flag & $4,00 \mathrm{a}$ & $0,00 \mathrm{a}$ & $2 \mathrm{a}$ & $5,00 \mathrm{a}$ & $0,17 \mathrm{a}$ \\
Acelga Fordhook Giant & $51,00 \mathrm{~b}$ & $10,5 \mathrm{~b}$ & $75 \mathrm{c}$ & $6,09 \mathrm{a}$ & $13,65 \mathrm{c}$ \\
\hline CV & 16,48 & 31,29 & 25,54 & 84,2 & 26,71
\end{tabular}

Letras diferentes en la misma fila denotan diferencias estadísticas según la prueba de Tukey, con un nivel de significancia del 0,05 \%.GE= germinación estándar; $\mathrm{PC}=$ primer conteo; $\mathrm{PE}=$ porcentaje de emergencia; $\mathrm{VG}=$ Velocidad de emergencia; IVG = índice de velocidad de emergencia. 
Los resultados obtenidos muestran que el porcentaje de germinación fue estadísticamente más alto $(\mathrm{P}<0,05)$ en la espinaca Viroflay con $66 \%$ de germinación, seguido de la acelga Foordhood Giant con 51 \%, mientras que los valores más bajos de germinación se observaron en los ajoporros American Flag y American Flag Large y en la espinaca New Zealand con valores de 9,4 y $0 \%$, respectivamente. Con relación a la velocidad de germinación, los híbridos que germinaron más rápido fueron la acelga Foordhood Giant y la espinaca Viroflay en 6,09 y 6,03 días, seguido de los ajoporros American Flag y American Flag Large, que germinaron a los 5,25 y 5 días, mientras que la espinaca New Zealand no germinó. El hibrido más eficiente durante la emergencia fue la acelga Foordhood con un IVE de 13,65, seguido de la espinaca Virofly, con IVE de 3,18. Por su parte, los valores más altos de IVE corresponden a ajoporros American Flag y American Flag Large, con 0,16 y 0,17.

La disminución del porcentaje de germinación estuvo asociada a la viabilidad de las semillas. En la Tabla 4 se observa que las semillas de ajoporros de American Flag, American Flag Large y espinaca New Zealand presentaron el menor porcentaje de germinación, obteniendo el menor número de semillas viables, mientras que las semillas de espinaca Viroflay y acelga Foordhood Giant, presentaron el mayor porcentaje de germinación y, a su vez, el mayor número de semillas viables al realizar la prueba de tetrazolio.

Tabla 4. Ensayo topográfico al tetrazolio de semillas de acelga, ajoporro y espinaca. Fuente: elaboración propia.

\begin{tabular}{ccc}
\hline Híbrido & \multicolumn{2}{c}{ Prueba de viabilidad al tetrazolio } \\
\cline { 2 - 3 } & S. Viables (\%) & S. No Viables (\%) \\
\hline Espinaca Viroflay & $59,50 \mathrm{~b}$ & $40,50 \mathrm{a}$ \\
Espinaca New Zealand & $6,25 \mathrm{a}$ & $93,75 \mathrm{c}$ \\
Ajoporro American Flag & $12,50 \mathrm{a}$ & $77,50 \mathrm{~b}$ \\
Ajoporro Large American Flag & $13,25 \mathrm{a}$ & $76,75 \mathrm{~b}$ \\
Acelga Fordhook Giant & $52,75 \mathrm{~b}$ & $47,25 \mathrm{a}$ \\
\hline
\end{tabular}

El alto porcentaje de semillas no viables en los materiales de ajoporros American Flag, American Flag Large, y de espinaca New Zealand se podría explicar por un inadecuado almacenamiento, lo que ocasiona oscilaciones de temperatura y humedad que afectan la viabilidad. Autores como [25] señalan que la mayoría de las especies hortícolas pierden rápidamente su viabilidad cuando los procesos de almacenamiento para su conservación no son controlados adecuadamente.

El bajo desempeño fisiológico en todos los materiales valuados, particularmente en espinaca New Zealand, ajoporro American Flag y ajoporro Large American Flag, pueden estar asociado a un tiempo prolongado de almacenamiento, así mismo como a las condiciones mismas del almacenamiento, como temperatura y humedad, lo cual conlleva a la pérdida de vigor y viabilidad de las semillas y al incremento de Aspergillus [26] y Penicillium en ellas [27]. En este orden de ideas, [28] reportaron la presencia de 11 tipos de hongos en semillas de espinacas, lo cual causa una drástica disminución del porcentaje de germinación.

Debido a la relación existente entre la frecuencia de patógenos y la disminución del desempeño fisiológico, se realizó la detección y cuantificación de la microbiota asociada a semillas de acelga, ajoporro y espinaca, cuyos resultados se observan en la Tabla 5. 
Tabla 5 Frecuencia de patógenos asociados a semillas de acelga, ajoporro y espinaca Fuente: elaboración propia.

\begin{tabular}{cccccccc}
\hline Hortalizas & $\begin{array}{c}\text { Aspergillus } \\
\text { spp. }\end{array}$ & A. niger & Rhizopus & Fusarium & Mucor & Penicillium & Bacterias \\
\hline Espinaca Viroflay & 9,63 & 9,50 & $9,00 \mathrm{a}$ & $7,00 \mathrm{a}$ & 12,50 & 15,88 & $13,88 \mathrm{bc}$ \\
Espinaca New Zealand & 9,00 & 9,50 & $9,00 \mathrm{a}$ & $8,88 \mathrm{a}$ & 10,00 & 7,00 & $3,25 \mathrm{a}$ \\
Ajoporro American Flag & 11,63 & 9,50 & $9,00 \mathrm{a}$ & $8,88 \mathrm{a}$ & 10,00 & 9,00 & $7,30 \mathrm{ab}$ \\
Ajoporro Large American Flag & 13,25 & 9,50 & $9,00 \mathrm{a}$ & $9,25 \mathrm{a}$ & 10,00 & 9,00 & $9,88 \mathrm{~b}$ \\
Acelga Fordhook Giant & 9,00 & 14,50 & $16,50 \mathrm{~b}$ & $18,50 \mathrm{~b}$ & 10,00 & 11,63 & $18,38 \mathrm{c}$ \\
\hline $\mathrm{P}$ & 0,6937 & 0,0773 & 0,0099 & 0,0109 & 0,406 & 0,1153 & 0,0031 \\
\hline
\end{tabular}

**P<0,05 para que la prueba de Kruskal \& Wallis permita comparaciones entre rangos.

La mayor incidencia del hongo Aspergillus se observó en ajoporros American Flag y Large American Flag; la de los hongos Aspergillus niger y Fusarium spp., se presentó en el material acelga Foordhook Giant; mientras que la del Mucor sp. estuvo en la espinaca Viroflay; finalmente, la frecuencia más alta de bacterias se encontró en la acelga Foorhood Giant.

Por su parte, la menor incidencia del patógeno Aspergillus sp. se observó en espinaca Viroflay, espinaca New Zealand y acelga Fordhood Giant; de igual forma, la incidencia más baja de los patógenos Aspergillus niger y Rhizopus se dio en espinaca Viroflay, espinaca New Zealand, ajoporro American Flag y ajoporro Large American Flag; con respecto al Fusarium sp., los valores más bajos se observaron en espinaca Viroflay; en relación con el Mucor sp., la menor incidencia correspondió a la espinaca New Zealand, ajoporro American Flag, ajoporro Large American Flag y acelga Fordhook Giant; mientras que los valores más bajos de Penicillium sp. se encontraron en espinaca New Zealand, y los de picnidio en espinaca Viroflay, ajoporro Large American Flag y acelga Fordhook Giant; finalmente, para bacteria, la menor incidencia se observó en espinaca New ZealandSi bien el patrón observado de los patógenos evaluados fue irregular, el comportamiento evidencia que todos los materiales evaluados tuvieron la presencia de patógenos en una mayor o menor proporción, lo que indica que las condiciones y tiempo de almacenamiento, particularmente la humedad y la temperatura, no fueron las adecuadas, lo que influyó negativamente en el desempeño fisiológico [29]. Debido a esto, la presencia de patógenos que afectaron el vigor y la viabilidad de las semillas, además de representar un riesgo en campo donde las condiciones ambientales externas pueden potenciar el desarrollo de estos patógenos [30], [31], y, si bien los hongos pueden crecer sobre varios sustratos, su desarrollo está relacionado con las condiciones de temperatura, humedad, iluminación, ventilación, disponibilidad de nutrientes, $\mathrm{pH}$ y contaminación atmosférica. No obstante, lo anterior, las condiciones del almacenamiento son un factor esencial en el desarrollo fúngico.

En este sentido, [32] afirman que la principal vía de propagación es la semilla botánica, y que la misma es afectada por diversos microorganismos, entre los que sobresalen los hongos. Estos investigadores encontraron, al evaluar el contenido de humedad, germinación y viabilidad en semillas de Leucaena leucocephala, que las semillas con menor tiempo de almacenamiento mostraron los mayores valores de germinación y viabilidad (71 \% y 93,5 \%), mientras que a los 18 meses fueron de $26 \%$ y 67,7\%, respectivamente. En este mismo estudio se identificaron 16 especies de hongos en la semilla: Aspergillus flavus, Aspergillus wentii, Aspergillus niger, Aspergillus sp., Alternaria alternata, Chaetomium indicum, Fusarium semitectum, Fusarium sambucinum, Fusarium oxysporum, Fusarium chlamydosporum, Fusarium incarnatum, Cladosporium sphaerospermum, Penicillium expansum, Rhizopus stolonifer, Trichoderma sp. y Pestalotia sp., los cuales afectaron desfavorablemente el 
desempeño fisiológico de las semillas, particularmente cuando el tiempo de almacenamiento fue mayor.

Para evitar la proliferación de patógenos en semillas es necesario que se cumpla rigurosamente con las medidas de almacenamiento adecuadas [33], particularmente las referidas a temperatura, humedad y tiempo de almacenamiento.

Así mismo, existen otros factores intrínsecos que pueden proteger el ataque de hongos y plagas en las semillas, como son la presencia de metabolitos secundarios en el interior de la semilla [34]; es por ello por lo que, adicional a las medidas fitosanitarias, es importante cuantificar la concentración de los principales metabolitos secundarios y su relación con la incidencia de patógenos en los materiales evaluados, la concentración de alcaloides flavonoides y fenoles para cada lote (Tabla 6).

Tabla 6. Concentración de metabolitos secundarios presentes en extractos etanólicos de semillas de hortalizas Fuente: elaboración propia.

\begin{tabular}{ccc}
\hline Hortalizas & Fenoles $\mathrm{mg} / \mathrm{ml}$ & Flavonoides $\mathrm{mg} / \mathrm{ml}$ \\
\hline Espinaca Viroflay & $93,32 \mathrm{~b}$ & $368,34 \mathrm{~d}$ \\
Espinaca New Zealand & $198,6 \mathrm{c}$ & $33,91 \mathrm{a}$ \\
Ajoporro American Flag & $43 \mathrm{a}$ & $109,21 \mathrm{~b}$ \\
Ajoporro Large American Flag & $0 \mathrm{a}$ & $112,16 \mathrm{~b}$ \\
Acelga Fordhook Giant & $163,78 \mathrm{c}$ & $261,78 \mathrm{c}$ \\
CV & 20,02 & 18,01 \\
\hline Significancia & $* *$ & $* *$
\end{tabular}

C.V.: coeficiente de variación; Prueba de Significancia: $n s: \mathrm{P}>0,05 ;{ }^{*}: \mathrm{P} \leq 0,05 ;{ }^{* *}: \mathrm{P}<0,01$.

Los valores más altos de fenoles se encontraron en las semillas de espinaca New Zealand con una concentración de 198,6 mg/ML, y acelga Fordhook Giant, con 163,78 mg/ML, seguido de espinaca Viroflay, quien presentó una concentración de 93,32 mg/ML, mientras que los valores más bajos se observaron en semillas de ajoporro American Flag con $43 \mathrm{mg} / \mathrm{ML}$ y ajoporro Large American Flag con valores de 43 y 0 mg/ML, respectivamente.

En lo que respecta a la concentración de flavonoides, los valores más altos se observaron en semillas de espinaca Viroflay con 368,34 mg/ML, seguido de acelga Fordhook Giant, con una concentración de 261,78 mg/ML; ajoporro Large American Flag, con 112,16 mg/ML y ajoporro American Flag, con 109,21 mg/ML, mientras que los valores más bajos se observaron en espinaca New Zealand, con concentración de 33,91 mg/ML.

En el caso de los fenoles se ha demostrado que pueden aumentar su concentración cuando las semillas se ven expuestas a condiciones adversas que comprometan su viabilidad [35]. Así mismo se evidenció que una mayor concentración de fenoles aumenta la viabilidad y longevidad de las semillas [36], en este caso las semillas con menor concentración de fenoles (ajoporro Large American Flag y ajoporro American Flag) tuvieron el peor desempeño fisiológico al tener los porcentajes de germinación y emergencia más bajos, lo cual es producto de la reducción de la viabilidad de las semillas, lo que se traduce en una menor longevidad y tiempo de vida útil de las mismas, mientras que las semillas con mayor concentración de fenoles (espinaca New Zealand y acelga Fordhook Giant) fueron las que tuvieron un mejor desempeño fisiológico y un mejor crecimiento de las plántulas.

\subsection{Análisis de los componentes principales}

Para estudiar el comportamiento de los 5 materiales evaluados y su comportamiento en función de su potencial fisiológico para garantizar la germinación y emergencia de las 
semillas, se realizó un análisis exploratorio de los datos a través de un análisis de componentes principales (ACP), cuyos resultados se observan en la Figura 4.

En la Figura 3 se observa que el 59,0 \% de la variación es explicado por el componente 1. En el biplot se evidencian dos grupos, unos asociados a un mejor desempeño fisiológico (espinaca Viroflay y acelga Foorhood Giant), los cuales poseen altos valores de germinación total y de porcentajes de flavonoides, mientras que el otro grupo (espinaca New Zealand, ajoporro American Flag y ajoporro Large American Flag) presenta un pobre desempeño fisiológico asociado al porcentaje de semillas muertas y frecuencia de picnidios que indica la presencia de hongos como Macrophomina phaseolin [2].

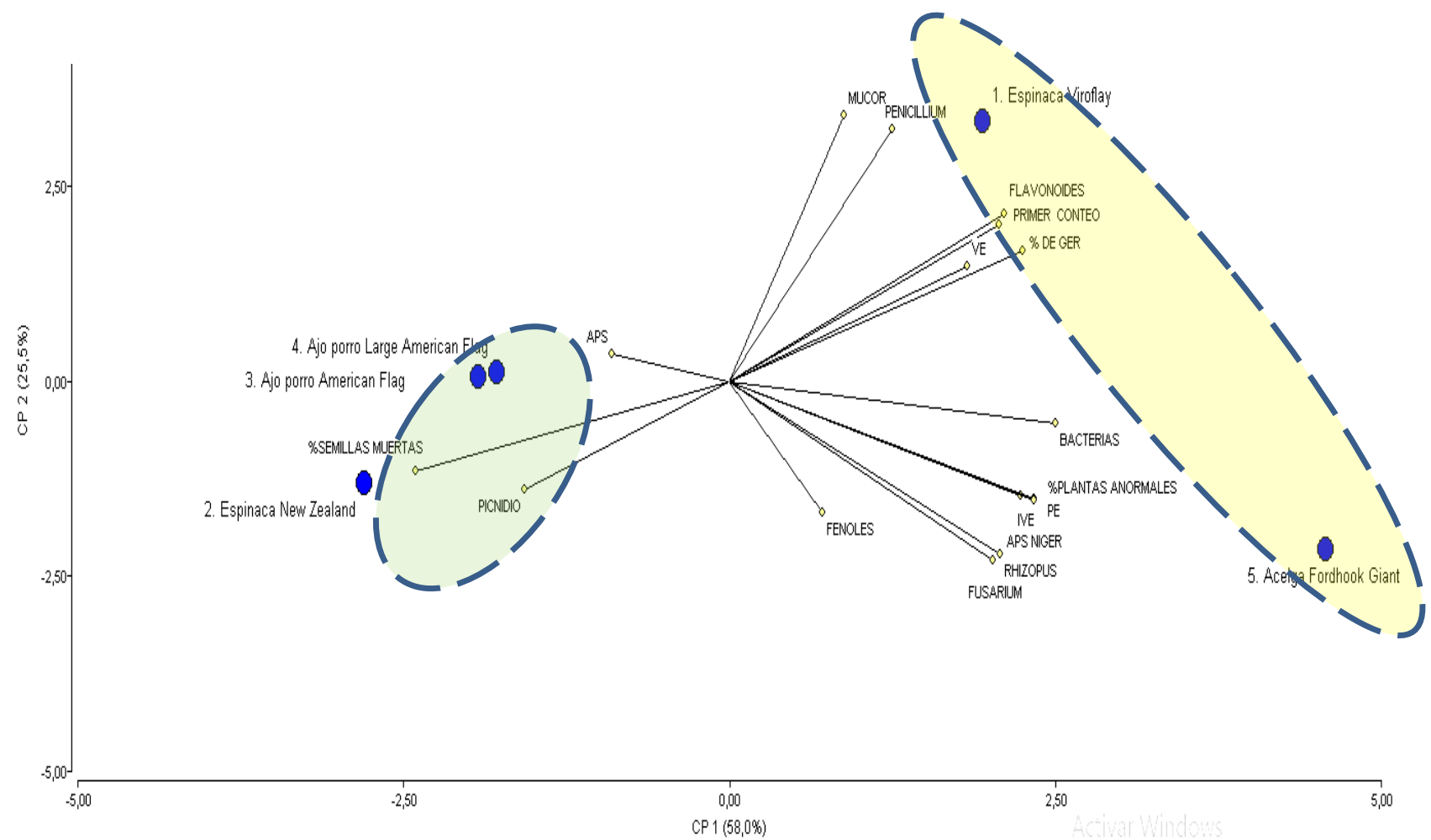

Figura 3. Biplot de análisis de componentes principales para el estudio del potencial fisiológico de espinaca Viroflay, espinaca New Zealand, ajoporro American Flag, ajoporro Large American Flag y acelga Fordhook Giant. Fuente: elaboración propia.

$\mathrm{Al}$ analizar el componente 2 que explica las variables, las semillas de acelga Foorhood Giant que presentan una mejor germinación y emergencia en comparación al resto de los materiales evaluados, se asociaron a una frecuencia elevada de Aspergillus sp., Aspergillus niger, Fusarium y Rhizopus; sin embargo, la alta concentración de fenoles permitió mantener un adecuado vigor y longevidad de las semillas a pesar de la presencia de patógenos. En el caso de la espinaca Viroflay se observó una asociación con los patógenos Mucor y Penicillium; si bien el alto contenido de flavonoides le ofreció protección a las semillas frente al ataque de los patógenos, estos resultados ratifican que los materiales con alto contenido de metabolitos secundarios mejoran las condiciones sanitarias de la semilla frente al ataque de patógenos; adicionalmente, el mejoramiento de las condiciones de almacenamiento aumentó el vigor de las semillas y redujo la incidencia de patógenos [37], [38].

Los resultados de esta investigación muestran que el desempeño físiológico de los materiales evaluados fue condicionado por la fecha de almacenamiento y las condiciones de almacenamiento que generaron situaciones inadecuadas de temperatura y humedad. 


\section{CONCLUSIONES}

Como resultado de lo expuesto en esta investigación, se establece que el desempeño fisiológico se ve afectado directamente sobre las variables de germinación, emergencia y velocidad de emergencia, sugiriendo un problema de viabilidad y vigor de las semillas producto de condiciones inadecuadas de almacenamiento.

La frecuencia de patógenos asociados a las semillas se evidencia por la presencia de varios patógenos, lo cual se relaciona con un mayor número de plántulas anormales y el inadecuado desempeño de las semillas evaluadas, particularmente los híbridos: espinaca New Zealand, ajoporro American Flag y ajoporro Large American Flag.

El mejor desempeño fisiológico de las semillas espinaca Viroflay y acelga Fordhook Giant estuvo asociado a un mayor contenido de flavonoides que ofrecen protección frente a la acción de patógenos y aumentan el vigor y la viabilidad de las semillas.

\section{AGRADECIMIENTOS}

Los autores agradecen al consejo de desarrollo humanístico y tecnológico de la universidad Lisandro Alvarado (CDCHT-UCLA) por el financiamiento parcial de la investigación a través de los fondos asignados en el programa de estímulo a la investigación Lisandro Alvarado (PEILA) en las ediciones 2019 y 2020 Colombia por su compromiso con incentivar el interés por la investigación científica.

\section{CONTRIBUCIONES}

Sorianny Álvarez-Orozco: desarrollo de la investigación en análisis de laboratorio.

Duilio Torres-Rodríguez: conceptualización, diseño y desarrollo de la investigación y en la redacción y revisión final del manuscrito (tutor académico),

Pastora Querales: asesor orientación en análisis fitopatológico y prueba de tetrazolio.

Rosario Valera: asesor en pruebas y ensayos de metabolito secundarios.

José Daniel Pacheco-Pacheco: desarrollo de la investigación en pruebas de germinación.

Tatiana Gavilánez: asesoramiento y revisión del manuscrito.

\section{CONFLICTOS DE INTERÉS}

No hay conflicto de interés que declarar por parte de los autores.

\section{REFERENCIAS}

[1] J. Di Noia; C. Byrd-Bredbenner, "Determinants of fruit and vegetable intake in low-income children and adolescents", Nutrition reviews, vol. 72, no. 9, pp. 575-590. Sep. 2014. URL

[2] L. Martínez; G. Ros; G. Nieto, "Hydroxytyrosol: Health benefits and use as functional ingredient in meat", Medicines, vol. 5, no. 1, pp.13. Jan. 2018. https://doi.org/10.3390/medicines5010013

[3] S. Ding; H. Jiang; J. Fang, "Regulation of immune function by polyphenols", J. Immunol. Res vol. 2018 , pp. 1-8. Apr. 2017. https://doi.org/10.1155/2018/1264074

[4] D. Aune et al., Fruit and vegetable intake and the risk of cardiovascular disease, total cancer, and all-cause mortality - a systematic review and dose-response meta-analysis of prospective studies", Int. J. Epidemiol., 
vol. 46, no. 3, pp. 1029-1056. Feb. 2017 https://doi.org/10.1093/ije/dyw319

[5] H. E. Khoo; A. Azlan; S. Teng Tang; S. Meng Lim, "Anthocyanidins and anthocyanins: colored pigments as food, pharmaceutical ingredients, and the potential health benefits", Food Nutr. Res., vol. 61, no. 1, Aug. 2017. https://doi.org/10.1080/16546628.2017.1361779

[6] R. Bulgari, "Studies of Quality and Nutrient use Efficiency in Vegetable Crops Grown under Different Sustainable Cropping System”. (Tesis de Doctorado), Dep. of Agricultural and Environmental Sciences, University of Milan. URL

[7] P. F. Scheelbeek et al., "Effect of environmental changes on vegetable and legume yields and nutritional quality”, Proc. Natl. Acad. Sci., vol. 115, no. 26, pp. 6804-6809. Jun. 2018. http://www.pnas.org/cgi/doi/10.1073/pnas.1800442115

[8] A. Garibaldi; G. Gilardi; S. Matic; M. L. Gullino, "First Report of Leaf Smut Caused by Entyloma gaillardianum on Gaillardia aristata in Italy", Plant disease, vol. 102, no. 3, pp. 852-862. Jan. 2018. https://doi.org/10.1094/PDIS-07-17-1092-PDN

[9] D. O. Chellemi; A. Gamliel; J. Katan; K. V. Subbarao,"Development and deployment of systems-based approaches for the management of soilborne plant pathogens", Phytopathology, vol. 106, no. 3, pp. 216-225. Feb. 2016. https://doi.org/10.1094/PHYTO-09-15-0204-RVW

[10] U. K. Vandana; A. Chopra; A. Choudhury; D. Adapa; P. B. Mazumder, "Genetic diversity and antagonistic activity of plant growth promoting bacteria, isolated from tea-rhizosphere: a culture dependent study", Biomedical Research, vol. 29, no. 4, 2018. https://doi.org/10.4066/biomedicalresearch.29-18-428

[11] S. Takahama et al., "Classification and Screening of Baby-leaf Vegetables on the Basis of TheirYield, External Appearance and Internal Quality", Hortic. J, vol. 88, no. 3, pp. 387-400. May. 2019. https://doi.org/10.2503/hortj.UTD-033

[12] D. Clavel, "Afrique: une politique semencière sous influence", Natures Sci. Sociétés, vol. 24, no. 2, pp. 168172. Apr. 2016. https://doi.org/10.1051/nss/2016016

[13] M. González de Molina; G. I. Guzmán Casado, "Agroecology and ecological intensification. A discussion from a metabolic point of view", Sustainability, vol. 9, no. 1, pp. 86. Jan. 2017. https://doi.org/10.3390/su9010086

[14] ISTA. "International seed testing association" Germination Sec, Chapter 19, pp. 19-41. 2016

[15] T., Bareke. "Biology of seed development and germination physiology", Adv Plants Agric Res, vol. 8, no. 4, pp. 336-346. Aug. 2018. URL

[16] A. R. Kermode, "Regulatory mechanisms in the transition from seed development to germination: interactions between the embryo and the seed environment", In Seed development and germination, 41, pp 273. 1995. URL

[17] R. Patil; R. Hunje; G. Somanagouda; S. S. Chadrashekhar. "Influence of seed enhancement techniques on seed quality in onion (Allium cepa L.)", Journal of Pharmacognosy and Phytochemistry, vol. 8, no. 6, pp. 252255. Oct. 2019. URL

[18] P. Kumar; S. Pathak; M. Kumar; P. Dwivedi, "Role of secondary metabolites for the mitigation of cadmium toxicity in sorghum grown under mycorrhizal inoculated hazardous waste site", In Biotechnological Approaches for Medicinal and Aromatic Plants, Springer, Singapore. pp. 199-212. https://doi.org/10.1007/978-981-13-0535-1_8

[19] J. B. Edmond; W. J. Drapala. "The effect of temperature, imersion in acetone and sulfuric acid on germination of five varieties of okra seed". Proceedings of the American Society for Horticultural Science, St. Joseph, vol. 74, pp. 601-606. 1959.

[20] P. Maguire, "From tree dwellings to new towns". Longmans. 1962. URL

[21] ISTA. "International seed testing association. International Rules for Seed Testing". (2009). Ed. Bassersdorf, Switzerland: ISTA, pp. 2-18.

[22] D. Marcano; M. Hasegawa, "Fitoquímica orgánica," Universidad Central de Venezuela, Consejo de desarrollo científico y humanístico, Caracas, Venezuela, pp. 29-59. URL

[23] J..A Di Rienzo.; F. Casanoves ; M. G. Balzarini; L. Gonzalez; M. Tablada; C. W. Robledo, InfoStat versión 2019. Córdoba: Grupo InfoStat, FCA, Universidad Nacional de Córdoba, Argentina. URL

[24] D. Liu; Y. Cui; R. Walcott; J. Chen, "Fate of Salmonella enterica and enterohemorrhagic Escherichia coli cells artificially internalized into vegetable seeds during germination". Appl. Environ. Microbiol., vol. 84, no.1, Oct. 2017. https://doi.org/10.1128/AEM.01888-17

[25] A. Kunova; M. Bonaldi; M. Saracchi; C. Pizzatti; X. Chen; P. Cortesi, "Selection of Streptomyces against soil borne fungal pathogens by a standardized dual culture assay and evaluation of their effects on seed germination and plant growth". BMC Microbiology, vol. 16, no.1, pp. 1-272. Nov. 2016. https://doi.org/10.1186/s12866-016-0886-1

[26] N. R. Gonçalves; S. M. Cicero; H. F. Abud, "Seedling image analysis and traditional tests to evaluate onion seed vigor”. Journal of Seed Science, vol. 39, no.3, pp. 216-223. Jul. 2017. https://doi.org/10.1590/23171545v39n3160444

[27] R. C. Mohanto; S. A. Shahriar; A. N. F. Ahmmed; F. A. Nishi; M. N. K. Eaty, "Prevalence of seed-borne 
fungi of different vegetables seeds in Bangladesh”, Research in Biotechnology, vol. 10, pp. 1-5. 2019. https://doi.org/10.25081/rib.2019.v10.5513

[28] P. Dube; P. C. Struik; E. Ngadze, "Seed health tests of traditional leafy vegetables and pathogenicity in plants”, Afr. J. Agric. Res., vol. 13, no. 15, pp. 753-770. Apr. 2018 https://doi.org/10.5897/AJAR2018.13001

[29] Z. U. Bashar; A. Wayayok; M. S. M. Amin; R. M. Mahadi, "Quality seed: an innovative sorting technique to sustainable, uniform and effective seedling establishment in nursery for system of rice intensification", $J$. Agric. Sci., vol 6, no. 7, pp. 185-193. http://dx.doi.org/10.5539/jas.v6n7p185

[30] W. S. Jansen van Rensburg; N. L. Zulu, A. S. Gerano; P. O. Adebola, "Seed production of African leafy vegetables: some experiences", in XXIX International Horticultural Congress on Horticulture: Sustaining Lives, Livelihoods and Landscapes $\quad$ (IHC2014). $\quad$ pp. https://doi.org/10.17660/ActaHortic.2015.1102.14

[31] N. Kumari; P. K. Rai; B. M. Bara; I., Singh, "Effect of halo priming and hormonal priming on seed germination and seedling vigour in maize (Zea mays L.) seeds," J. Pharmacogn. Phytochem., vol. 6, no. 4, pp. 27-30. 2017. URL

[32] A. A. M. Abd-Allah; N. A. M. N. El-Deen; W. A. M. Mohamed; F. M. Naguib, "Mast cells and proinflammatory cytokines roles in assessment of grape seeds extract anti-inflammatory activity in rat model of carrageenan-induced paw edema”, Iran. J. Basic Med. Sci., vol. 21, no. 1, pp.97-107. Jan. 2018. https://doi.org/10.22038/IJBMS.2017.25067.6219

[33] J. C. Lezcano; M. Navarro; Y. González; O. Alonso, "Determinación de la calidad de las semillas de Leucaena leucocephala cv. Perú almacenadas al ambiente", Pastos y Forrajes, vol. 30, no. 1, pp. 1-10. URL

[34] J. S. Ninganna; B. S. Vyakaranahal, "Influence of Desiccants on Quality of Onion Seeds during Ultra Dry Storage", Int. J. Curr. Microbiol. App. Sci, vol. 7, no. 12, pp. 1981-1987. 2018. https://doi.org/10.20546/ijcmas.2018.712.228

[35] S. Pagare; M. Bhatia; N. Tripathi; S. Pagare; Y. K. Bansal, "Secondary metabolites of plants and their role: Overview. Current", Trends in Biotechnology and Pharmacy, vol. 9, no. 3, pp. 293-304. URL

[36] V. Kumar, V. Tomar, S. A. Ranade, H. K. Yadav \& M Srivastava, "Phytochemical, antioxidant investigaations and fatty acid composition of Lepidium sativum seeds", J. Environ. Biol., vol. 41, no. 1, pp. 59-65. 2020. http://dx.doi.org/10.22438/jeb/41/1/MRN-1136

[37] E. R Marques; R. F. Araújo; E. F. Araújo; S. Martins Filho; P. C. Soares; E. G. Mendonça, "Dormancy and enzymatic activity of rice cultivars seeds stored in different environments". J. Seed Sci, vol, 36, no. 4, pp. 435-442. Dec. 2014. https://doi.org/10.1590/2317-1545v36n41031

[38] P. Singariya; K. K. Mourya; P. Kumar, "Estimation of Antibacterial Efficacy in Alkaloids of Anogeissus rotundifolia an Indigenous Medicinal Plant against Some Pathogenic Micro-organisms", Asian J. Res. Chem, vol. 11, no. 2, pp. 432-440. 2018. https://doi.org/10.5958/0974-4150.2018.00079.2 